\title{
Sexual selection of conspicuous consumption
}

\author{
Vaios Koliofotis ${ }^{1}$ (D)
}

Accepted: 28 October 2021 / Published online: 23 November 2021

(c) The Author(s) 2021

\begin{abstract}
Recently, a number of papers draw upon ideas from sexual selection and costly signaling theory to argue that conspicuous consumption has evolved as a sexually selected mating strategy. I outline what are considered to be the criteria for arguing that a trait is the outcome of sexual selection and I explore whether conspicuous consumption is sexual adaptation. Though I share the insight that evolutionary theory can contribute to our understanding of consumption behavior, I argue that existing evolutionary explanations of conspicuous consumption do not examine human evolved psychology and available evidence about past environments. I further argue that cultural evolution theory provides an alternative explanation of conspicuous consumption in modern environments. In particular, conspicuous consumption is understood as a pattern of behavior marked by specific social learning mechanisms. Such an approach reflects the analytical tools of cultural evolution theory and provides a classification of cognitive factors involved in consumption choices.
\end{abstract}

Keywords Conspicuous consumption; consumer learning $\cdot$ Sexual selection $\cdot$ Costly signaling

JEL classification $\mathrm{A} 12 \cdot \mathrm{B} 15 \cdot \mathrm{B} 52 \cdot \mathrm{D} 01 \cdot \mathrm{D} 11 \cdot \mathrm{O} 12$

\section{Introduction}

Conspicuous consumption is one of the most striking phenomena in recent human history. Thorstein Veblen (1899) used the concept to bring forward original and provocative insights about the relation between consumer behavior and social status. Whereas Veblen focused on the elite of the middle to late nineteenth century western societies, conspicuous consumption is not limited to particular social groups but has an enormous diffusion to a vast part of contemporary human population. Over

Vaios Koliofotis

vkoliofot@hotmail.com

1 Erasmus Institute for Philosophy and Economics, Erasmus University Rotterdam, Burgemeester Oudlaan 50, 3062 Rotterdam, PA, Netherlands 
the past decades, consumers belonging in different economic, religious, ethnic and linguistic groups are increasingly engaged in conspicuous consumption.

Has conspicuous consumption evolved? What are the psychological mechanisms that underlie conspicuous consumption? And can we relate human economic decisions to the evolution of a culture that rewards conspicuous consumption?

Answering these questions is a daunting task. Sociological, biological, psychological and economic factors are deeply intertwined in the process of conspicuous consumption and it is difficult to completely isolate them from each other. Moreover, conspicuous consumption is a phenomenon driven by social interactions embedded in cultural groups, socioeconomic classes and communities in different regions and countries. Hence, it is hard to assert the primacy of particular causal factors.

A growing body of research attempts to explain consumer behaviors by applying evolutionary insights and methods (Miller 2001 and 2009, Saad 2014, Witt 2017). The current trend in the evolutionary literature is to explain conspicuous consumption by applying sexual selection and costly signaling theory. A number of papers argue that conspicuous consumption has evolved as a sexually selected mating strategy that provides reproductive benefits to males (e.g. Zak and Park 2002, Griskevicius et al., 2007, De Fraja 2009, Nelissen and Meijers 2011, Sundie et al. 2011, Collins Baer and Weber 2015). Like peacocks' large and colorful tails, conspicuous consumption impedes males' chances of survival while it confers mating benefits.

In what follows, I examine the arguments put forward by these evolutionary researchers and I identify areas of controversy that may spark a debate among economists, psychologists and evolutionary biologists. Given that evolutionary theory can contribute to our understanding of consumption practices, ${ }^{1} \mathrm{I}$ argue that in the recent literature evolutionary researchers attempt to examine the evolutionary underpinnings of conspicuous consumption without analyzing evidence about the ancestral environment, human psychological characteristics or socio-cultural parameters. In particular, an evolutionary explanation of conspicuous consumption based on sexual selection and costly signaling theory faces three problems:

- First, there is the issue of defining conspicuous consumption: Does this trait refer to psychological capacities that we have or certain types of behavior in which we engage?

- Second, there is the issue of whether conspicuous consumption has evolved as a sexually selected mating strategy: Conspicuous consumption does not conform to the conditions for the reliability of costly signaling traits.

- Third, there is an issue of the type of evidence needed to support an evolutionary explanation based on sexual selection and costly signaling theory: Available ethnographic evidence from foraging societies cast doubt whether conspicuous consumption evolved as a mating strategy.

\footnotetext{
1 The analysis of this paper is based on the idea that postulating evolutionary explanations has been successful in evolutionary biology and therefore evolutionary theory can have a heuristic value in the social sciences. Economists can reflect on evolutionary processes and the demands of past and present environments to better understand human cognition and economic behavior.
} 
Given these problems, it is worth exploring alternative theories that could explain the evolution of conspicuous consumption. In particular, cultural evolution theory provides a plausible way to study consumption behaviors in modern environments. One answer to the question of why people engage in conspicuous consumption involves cultural transmission: people observe and learn the behaviors of others during social interactions. The implication is that conspicuous consumption does not evolve as a display to potential mates but as a result of pre-existing evolved psychological traits.

Cultural evolution theory provides a framework that allows researchers to identify and classify the psychological biases that underlie conspicuous consumers. In contrast to an explanation based on sexual selection and costly signaling theory that focuses on displays that attract potential mates, I suggest that our psychological learning biases have a crucial motivating role in consumption decisions, including conspicuous consumption. These biases are genetically transmitted traits that influence the acquisition and retention of conspicuous consumption in the population.

Some clarifications are in order. Cultural evolution theory has not made any major inroad into economics, despite its growing representation in contemporary evolutionary research. Given the novelty of applying this account to the study of economic behavior, I focus on the role of social learning biases in consumers' decisions. However, the claim of this paper is not that the list of psychological biases I will present is exhaustive. Neither do I suggest that there are no other theoretical frameworks that could explain why we conspicuously consume. Instead, the aim is more modest: To present an evolutionary account that can facilitate future research on conspicuous consumption.

The paper is structured as follows. In the next section I provide a brief and general introduction to sexual selection and costly signaling theory, focusing on the aspects I consider more relevant for the study of conspicuous consumption. In section 3, I examine what kind of traits evolutionary researchers have in mind by conspicuous consumption. Section 4 explores in more depth whether conspicuous consumption does in fact possess the main features of sexual adaptations. Section 5 deals with evolutionary history and the importance of evidence about the ancestral environment to support hypotheses about sexual adaptations. Section 6 explores areas of research where cultural evolution can provide an original contribution with its commitment to develop hypotheses informed by psychology and evolutionary theory. I focus on cultural transmission biases that influence the propagation of conspicuous consumption in modern environments. Section 7 concludes.

\section{Sexual selection and costly signaling theory}

A number of recent papers draw upon ideas from sexual selection and costly signaling theory to argue that conspicuous consumption is a sexual adaptation (Griskevicius et al. 2007, Miller 2009, Fraja 2009, Nelissen and Meijers 2011, Sundie et al. 2011, Collins, Baer and Weber 2015). In what follows, I briefly describe how these theories explain the evolution of human and animal traits. 
Sexual selection is often invoked to explain the evolution of exaggerated costly traits. It is an evolutionary process that results in differential mating success and can generate rapid genetic and phenotypic change (Darwin 1871, Maynard-Smith 1978). Sexual selection comes in two main forms: a) intrasexual selection by competition (usually among males) and b) intersexual selection by (usually female) choice. Intrasexual selection leads to exaggerated traits that promote the reproductive success in species whose members of the same sex compete for access to mates of the other sex. A classic example is the large antlers of male red deer that repel rivals and allow access to territory and female herds. In intersexual selection, members of one sex choose as a mate a member of the opposite sex. This leads to the evolution of traits that make an organism more attractive as a mating partner for the members of the opposite sex.

A key feature of sexual selection is that it can lead to the evolution of a trait that has a negative influence on an organism's survival. In particular, females might choose traits that are detrimental to the males that have them and pass them on to the next generation. However, those females that prefer males that do not have these negative traits will produce offspring that live longer and will have more sexual interactions. Hence the challenge for evolutionary research is to provide an evolutionary explanation of why such extreme traits do better than more moderate traits.

A substantial literature on animal behavior proposes the following solution to this problem (Zahavi 1975, Grafen 1990a, b, Bliege Bird and Smith 2005). Some extravagant traits are good indicators of particular male qualities that females find important in their choice of a mating partner. The relation between the expression of a relatively costly male trait and particular male qualities is such that only higherquality males can afford to produce it. Hence, a female can use this trait as an indicator of a male's genetic or phenotypic qualities. Females benefit if they choose males who display an extreme trait, while males compensate the cost of the display by an increase in mating opportunities.

In the evolutionary explanation presented in the previous paragraphs it is possible to distinguish the following traits:

i) A male display trait, a costly quality-dependent signal emitted by human males;

ii) Male quality trait(s) indicated by the display trait;

iii) A female's preference ${ }^{2}$ for males with better quality traits. Since male quality traits are often unobservable, females prefer males with higher levels or better quality of the display trait;

Evolutionary researchers that apply costly signaling theory present the following conditions for a trait to qualify as a costly display (e.g. Griskevicius et al. 2007, Nelissen and Meijers, 2011):

\footnotetext{
${ }^{2}$ To keep the analysis simple, I assume that a female's preference for a mating partner coincides with her mating choice. This is not the case when there is competition among females to attract mates and males demonstrate a preference for a female with certain characteristics (e.g. resources, attractiveness).
} 
i) it must be easily observable by others;

ii) it must be hard to fake because of its associated costs to the signaler in terms of economic resources, time, energy, risk, whereby the costlier the trait the more likely it is to be an honest indicator;

iii) it must result in a fitness benefit for the male signaler such as an increased access to desirable female mates;

iv) it must be an indicator to potential female mates of some unobservable but desirable male trait or characteristic, such as access to resources, prosocial disposition, courage, physical health or intelligence;

The evolutionary argument is that human conspicuous consumption has these four features and therefore it is a sexual adaptation. Hence, there are two ways to criticize an evolutionary explanation of conspicuous consumption based on sexual selection and costly signaling theory. First, it is possible to question the claim that conspicuous consumption does have these four characteristics. Second, one can demonstrate that there is an alternative explanation that is more plausible from an evolutionary perspective. In the sections that follow I apply these two lines of argumentation. But before I present my arguments, it is important to clarify what kind of trait these researchers have in mind when they use the term conspicuous consumption.

\section{What is the main trait in question?}

One of the main problems of this literature is that it is often hard to understand what kind of trait do evolutionary researchers refer to by conspicuous consumption. A close reading of evolutionary models and empirical research suggests that a single term covers different concepts and processes across many disciplines. In this section I will examine in more detail evolutionary explanations based on sexual selection and costly signaling theory in an attempt to clarify the main trait under investigation.

In the presentation of a model inspired by costly signaling theory, Fraja (2009) states that it is the psychological desire for conspicuous consumption that is designed by sexual selection (p.62). For Collins, Baer, \& Weber (2015) conspicuous consumption refers to a male preference that has deep evolutionary roots. Yet, in other parts of his paper, Fraja (2009) defines conspicuous consumption as "consumption for its own sake" that "is easy to observe and expensive to acquire" (p.52). In Collins, Baer, \& Weber (2015) conspicuous consumption includes "any consumption activities beyond those required for survival" such as "developing art or other objects of beauty in traditional societies, or participating in the labour force to earn income in modern times" (p.191). Here it is assumed that a minimum level of consumption is necessary for survival (e.g. health care, food, housing) and all male activities that do not contribute to survival (e.g. entertainment, travelling, painting) are conspicuous consumption. Griskevicius et al. (2007) and Sundie et al. (2011) identify themselves as evolutionary psychologists and argue that in their experimental study they investigate the design features of psychological adaptations (e.g. 
Griskevicius et al., 2007: p.99). Their research focuses on some sort of psychological features predicted by costly signaling theory.

What can we conclude from this multitude of different formulations of conspicuous consumption? Conspicuous consumption is often treated as a psychological trait that is the target of sexual selection. In evolutionary research stemming from economics, conspicuous consumption refers to individuals' subjective motivations (desires, intentions or preferences) that underlie behavior. A closely related understanding of conspicuous consumption in evolutionary psychology refers to actual causal mechanisms that produce behavior. What sexual selection explains is not human behavior but the behavior generating mechanisms that we have.

The key issue with these alternative formulations of conspicuous consumption is that they are insufficiently clear to allow an in-depth understanding of the trait under investigation. Evolutionary researchers frequently use the term psychological mechanism to refer to everything from input-output information processes to subjective mental states involved in conspicuous consumption. Although it is common for evolutionary psychologists and economists to examine the design characteristics of our brain system, in this literature they are not explicit about the model of human cognition and psychology they apply. Does our brain contain specialized mechanisms or "modules" that evolved to solve fitness-relevant problems in the ancestral environment? Or is our brain a general-purpose problem solving device? It is not clear whether many of these conceptions of conspicuous consumption are rivals or they complement each other.

A distinct formulation is often used by evolutionary modelers to analyze conspicuous consumption. In evolutionary theory, conspicuous consumption is understood as behavior in relation to fitness outcomes, independently of the actor's conscious or subjective motivations. What sexual selection explains is why organisms acquired the behaviors that they have in a particular environment. For example, in costly signaling theory behaviors and behavioral strategies are often described as adaptive traits. What these models examine is fitness consequences of types of behaviors that are present in the human population.

The upshot is that there are different ways to think about conspicuous consumption that diverge from one another at the conceptual level. To understand the phenomenon of conspicuous consumption, it is essential to disentangle behavioral outcomes from psychological aspects. Conspicuous consumption might refer to a kind of a) internal behavior-generating mechanism or b) overt behavior produced by a mechanism. Hence, it is important for researchers to be clear about what exactly is the main trait they examine in their studies. Do they suggest that conspicuous consumption is a type of behavior that requires an evolutionary explanation? Or is conspicuous consumption is a psychological trait that interacts with the environment to produce a particular behavior?

In the analysis that follows I consider conspicuous consumption as a type of behavior. Evolutionary theorists rarely describe their models as comprising psychological mechanisms. However, these evolutionary models of behavior and strategies are often applied to generate testable hypotheses about human psychology.

In the sections I follow this line of argumentation: first, I examine whether the evolutionary explanations of conspicuous consumption meet the conditions specified 
in the literature and second, I analyze the type of evidence needed to support an evolutionary explanation based on costly signaling theory and sexual selection.

\section{Conspicuous consumption and costly signaling theory}

Whatever traits different researchers have in mind, they have to actually possess the four defining features presented in Section 2. In order for evolutionary researchers to support their argument, they must offer evidence that conspicuous consumption is an observable, costly trait that indicates particular qualities and provides fitness benefits to those that possess it. In the following sub-sections I argue that there are serious problems with this claim.

\subsection{Conspicuous consumption is observable}

Conspicuous consumption is by definition an observable trait. The first step in experimental research is to demonstrate that conspicuous consumption varies among the male members of a population. However, one of the major difficulties is that it is not sufficient to show that there is variation in conspicuous consumptions. Evolutionary researchers must also demonstrate that differences in male conspicuous consumption are perceived by female conspecifics. In other words, it must be shown that members of the female sex can discriminate between different rates or intensities of conspicuous consumption. Merely demonstrating that conspicuous consumption is observable is of no use unless it is possible to show that there is variation in this trait and that there is a consistent female preference for a specific part of the variation of conspicuous consumption.

This brings me to the following point: Conspicuous consumption behavior needs to be examined in a reproduction or mating context. For sexual selection, there must be some evidence that conspicuous consumption elicits female choices or psychological responses that are consistent with the possibility of conception. In other words, conspicuous consumption has to be experimentally tested at a time when the perception of the display can lead to successful reproduction. Hence, tests with university students (Griskevicius et al. 2007, Sundie et al. 2011) are useful only if it can be also shown that the same display have a similar effect when females make decisions about conception later in life. Especially in western societies where most experiments take place, it is a common practice for young couples to go through a prolonged period of their relationship where sex is not intended for conception. Female responses to conspicuous consumption during this period may not be informative about sexually selected displays when conception is intended.

\subsection{Conspicuous consumption is costly}

The high-cost criterion is commonly cited among evolutionary researchers. We have seen in section 2 that costs of sexual adaptations are of central importance to the theory of sexual selection and costly signaling theory. In the evolutionary models 
of Fraja (2009) and Collins, Baer and Weber (2015), costs have been explicitly described as having a detrimental influence on the survival of those that conspicuously consume. If fitness is measured in terms of offspring produced throughout the lifetime of an individual, then to count as an evolutionarily significant cost, an increase of conspicuous consumption has to lead to a decrease in survivability of males and an increase in the number of offspring they produce.

In the experimental literature, however, there is often confusion over what qualifies as a cost. Typically, costs refer to expenditure of resources such as money, time, energy or risk, whereby the costlier the trait the more likely it is to be an honest display. (Graskevicious et al. 2007, Nelissen and Meijers, 2011, Sundie et al. 2011). Conspicuous consumption is often associated with the purchase of expensive products that are consumed in public and are used by males to display material wealth. However, it is important to make a distinction between resource expenditure and fitness costs: conspicuous consumption may require a high expenditure of money or time without being costly in terms of lifetime male survivability. The claim that conspicuous consumption is costly relies almost exclusively on indirect evidence about resource expenditure while empirical evidence on the actual influence on lifetime reproduction success is at best circumstantial.

There is an additional problem with the handicap principle frequently cited by researchers as a mechanism for ensuring that conspicuous consumption is a reliable trait. The costly signaling hypothesis suggests that the cost associated with the production or maintenance of the display is crucial for its reliability. However, recent research on costly signaling theory suggests that a decrease in a fitness component is not necessary to prevent deception (Grose 2011, Számadó 2011). In other words, a high display cost is not the only mechanism that maintains honesty and cheap or even cost-free displays can also be honest and reliable. For example, according to the index hypothesis, the actual fitness costs of honest displays could be zero. A display that is not difficult to produce or maintain can still be honest if there is causal link between a male quality (e.g. material wealth) and the display (e.g. conspicuous consumption) (e.g. Dezecache, Mercier and Scott-Phillips 2013). Cheap or even cost-free signals can also be honest although they have largely been ignored in the research of conspicuous consumption.

To sum up, in the recent literature fitness costs are confused with expenditure. Costs in terms of resources are suggested to provide evidence for the fitness costliness of conspicuous consumption. In line with theoretical requirements, more care is needed in future empirical studies to distinguish fitness costs from resource expenditure. In addition, evolutionary researchers working on conspicuous consumption must justify their preference of particular evolutionary models over other alternatives that provide evolutionary explanations of why conspicuous consumption is a reliable display.

\subsection{Conspicuous consumption provides fitness benefits}

The third criterion is that conspicuous consumption must increase the probability that the male signaler will gain some fitness advantage through the display, such as 
increased ability to attract and copulate desirable mates. This is not easy to experimentally test in human populations, mainly because it is difficult to demonstrate that those that have a display trait produce more offspring through their lifetime. There are no studies that show a correlation between male conspicuous consumption and differential reproductive benefits. At best, there is plenty of anecdotal evidence that human females find male conspicuous consumers attractive. In the absence of empirical studies that show that differences in conspicuous consumption actually results in differential reproductive success, a claim that conspicuous consumption is a sexual adaptation is simply a hypothesis in need for empirical support.

There is an additional and more serious problem in the claim that conspicuous consumption is a sexual adaptation. Empirical studies in evolutionary psychology suggest that human females prefer high status males or those that demonstrate the potential to attain high status (Buss 1989 and 2016, Geary 1998). Using this insight, evolutionary researchers often argue that display traits such as conspicuous consumption are used by human males to maintain or increase their status. This argument finds conspicuous consumption to have an intermediary role: an increase in conspicuous consumption leads to an increase in a male's status relative to the status of other competitors. Females prefer higher status mates and therefore there is an increase in male reproductive success.

What is problematic with this argument is that in this literature it is also assumed that conspicuous consumption has negative or minimal influence on survivorship (Fraja, 2009, Collins Baer and Weber 2015). The pursuit of social status via conspicuous consumption however can provide direct or indirect benefits for high status males in addition to mating access. Such benefits include deference, coalitions, aid in childcare or after injury and illness, leniency after a failure to reciprocate. The benefits derived from having status can be cumulative since they are received from multiple conspecifics. Hence the overall impact of high status on fitness is not only an increase in fertility, but also an increase in the viability (or well-being depending on how viability is conceived) of self, spouse, offspring and other kin.

\subsection{Conspicuous consumption is an indicator of male quality traits}

By their very construction, evolutionary models of conspicuous consumption treat all male qualities traits as exogenous. Females are assumed to have a fixed preference for whatever male qualities are displayed (Collins Baer and Weber 2015: p.194). Moreover, quality traits are not heritable but are randomly allocated at birth. These assumptions allow tractable models for the theoretical analysis of conspicuous consumption (Fraja 2009, Collins Baer and Weber 2015: p. 203). However, this also creates ambiguity about what kind of attributes males convey by the display trait. Accumulated resources (i.e. wealth), potential to acquire resources, dexterity, altruism, courage, health, status, physical condition, artistic skills, ambition, industriousness, intelligence or (unspecified) genetic qualities are mentioned in this literature as quality attributes indicated by conspicuous consumption. Whether there is a reliable link between a males' conspicuous consumption and these qualities cannot simply be assumed to be true but require experimental and theoretical investigation. 
A plausible response to this problem is the following. It is possible to argue that females choose mates that conspicuously consume because this is a good indicator of a male's overall quality. When it is difficult to determine specific quality traits of potential mating partners, a female can use conspicuous consumption as a proxy of a male's composite of genetic or phenotypic qualities. Whether this hypothesis is plausible depends on the environment in which mating interactions are taking place. For example, in small and stable groups, females would not be so much interested in males' conspicuous consumption because they could acquire information about specific qualities of potential mates through gossip or repeated interactions.

To conclude this section, it is doubtful whether conspicuous consumption meets the criteria of costly signaling theory and sexual selection. As we will see in the next section, problems only multiply when one considers that the male display trait and female preference are supposed to have evolved sometime during the Pleistocene.

\section{Conspicuous consumption and evidence from hunter-gatherer societies}

In order for evolutionary researchers to make their argument they must offer reasonable evidence that conspicuous consumption does in fact possess the characteristics presented in the previous section. We have seen that there are reasons to doubt this claim. Suppose however that evolutionary research has experimentally demonstrated that conspicuous consumption is an easily observable, costly trait that confers reproductive success to males. Let us suppose even further that evolutionary research has shown that females have a preference for males' level or quality of conspicuous consumption. A question left to ask is whether these researchers have shown that conspicuous consumption is a sexual adaptation.

Most of the published literature implicitly assumes that to demonstrate that a trait such as conspicuous consumption has the characteristics presented in section 2 is sufficient to conclude that a trait evolved by sexual selection. In current research there is little effort in establishing a plausible link between the display trait, the quality traits and the preference traits by using available information about the ancestral environment. It appears that evolutionary research has lost track of the kind of evidence is needed for the empirical support of sexual selection explanations. Even if evolutionary researchers demonstrate that conspicuous consumption has the characteristics presented in the previous section, one has no reason to think that it is likely brought about by sexual selection. At best, evidence about current behavior and current mating success can only suggest that sexual selection acts in the present environment. No matter which traits one chooses to examine, some evidence about the ancestral environment is essential to support hypotheses about sexual adaptations.

This critique goes back to the classic work of Gould and Lewontin (1979). However, the key concern here is not so much that an evolutionary explanation of conspicuous consumption is not well supported by empirical evidence. The point is that historical evidence complements evolutionary explanations and is important in our understanding of the trait under investigation. If researchers claim that conspicuous consumption is a sexual adaptation in a particular environment, it is essential 
to present in some detail what that environment is like. Conspicuous consumption needs to be understood according to its unique, time and place in human evolution. Some evolutionary researchers are aware of the present-centered starting point of their investigation but think that knowledge of how sexual selection is operating in the present allows them to extrapolate back in time. This, however, is problematic unless one can also demonstrate that the selection conditions have remained constant through time. In turn, examining whether this assumption holds requires evidence about our ancestral past.

Fraja (2009) seems to agree and suggests that conspicuous consumption "was hard wired in the brain of early humans prior to their dispersion from Africa, and therefore must have provided evolutionary advantages in the conditions prevailing between one million and 80,000 years ago" (p.52). In order to get antecedent knowledge about the social organization, mating patterns and food acquisition in the ancestral past, anthropologists and ethnographers typically examine present huntergatherer societies not conducive to agriculture. ${ }^{3}$ These foraging groups might have changed significantly over time but they can shed light on the social environment in which the evolution of human traits has taken place.

What can we infer about our distant ancestors by looking at the few thoroughly studied hunter-gatherer societies of recent times? Ethnographers that study modern foraging groups as a proxy of ancestral hunter-gatherer societies have arrived at some generally accepted conditions about Pleistocene social life:

i) Population density was low and groups were small with an average size of a few hundred members ${ }^{4}$;

ii) Groups of hunter-gatherers were highly mobile and nomadic, moving frequently from one territory to another to get food resources necessary for subsistence;

iii) There was little difference in resources among individuals because sharing practices were common;

If we accept that hunter-gatherer populations have the above characteristics, there are two important implications for an evolutionary explanation of conspicuous consumption based on costly signaling theory and sexual selection.

\footnotetext{
3 There is a great diversity among these groups (Marlowe, 2005, Kelly 2007, Henrich and McElreath 2007) and anthropology typically distinguishes between delayed-return and immediate-return huntergatherer societies (Woodburn 1982). Immediate-return societies are considered a good proxy of life in the human past while more modern delayed-return societies demonstrate marked inequalities, social hierarchy and larger group size. As any other categorization, it is not absolute; in some groups, it is possible to find characteristics of both delayed and immediate-return societies. The evidence examined in this paper is based on immediate-return hunter-gatherer societies.

4 Ethnographic research demonstrates that hunter-gatherer populations operate in a network of groups that have different sizes. Small bands with a size of around thirty five individuals are embedded into larger groups of a few hundred members (e.g. Kelly 2007, Gamble 1999, Marlowe 2005).
} 
First, a display trait such as conspicuous consumption would have little value in small and relatively stable groups, ${ }^{5}$ either because there is continuous interaction between group members or because private information would have become public through third parties that communicate the quality traits of group members. In small hunter-gatherer groups, it is more likely that mate choice is based on the accumulation and integration of information over a long period of time through repeated encounters or over the course of development. ${ }^{6}$

More generally, females have an incentive to respond to a display trait such as conspicuous consumption when they face information asymmetry about male private quality traits such as accumulated resources, status, skills related to the acquisition of food or the construction of privately owned objects. Because there is female uncertainty about these traits, males are motivated to conspicuously consume and females value this display trait. In the context of foraging societies, demographic factors such as small group size mitigate information asymmetry and the value of a display trait such as conspicuous consumption.

Second, these nomadic hunter-gatherer groups strive to meet daily subsistence requirements without devices that allow the storage of a surplus in resources. They often emphasize sharing in the distribution of resources and reciprocation to endure harsh environmental conditions. The implication of ethnographic research is that even if there is inequality in hunting abilities, there is near equality in lifetime consumption of food resources. When the prey is not consumed near the place where it is captured, specific sharing rules prescribe how it is distributed among the nuclear family and other member of the group. Moreover, displays of wealth cannot be used as source of information in a mating context because storage of accumulated resources is nonexistent. In contrast to modern societies, in these hunter-gatherer groups inequality in the production of resources (i.e. capturing game) does not result to major inequalities in accumulated resources (i.e. wealth) or consumption of resources.

Since sharing norms and the nomadic lifestyle prevent saving and accumulation of resources, it leaves the production and possession of skillfully made objects (i.e. tools, weapons, ornaments) to act as a signaling medium directed to potential mates. Collins, Baer, and Weber (2015: p.191) and Fraja (2009; p.62) suggest that the work of Kohn and Mithen (1999) on the elaborate morphology of Palaeolithic Acheulian handaxes supports the hypothesis that conspicuous consumption is a sexual adaptation. According to Kohn and Mithen (1999), impractically large hand axes made

\footnotetext{
5 The boundaries of hunter-gatherer groups are often fluid and local bands fission or fuse in search of resources (Marlowe, 2005). If the group size is very small, it will require regular genetic inputs from other camps in order to stay viable. This, however, does not necessary implies that when males move to other camps they engage in conspicuous consumption to attract mating partners that belong to other bands. I thank Krist Vaesen for bringing these points to my attention.

${ }^{6}$ Hunter-gatherers appear to promote play from a very young age in order for children to acquire the skills, knowledge and values of their group (e.g. Gray 2014). Until they reach reproductive maturity, females have ample opportunity to observe the skills of potential mates that will become successful adults in their group.
} 
from about 1.4 Myr ago to around 100,000 years ago were symbols of status, built and displayed by males to attract potential mates.

However, providing an evolutionary explanation of why these hand axes have been built is one of the most controversial research topics in Palaeolithic archaeology. After decades of research, it is still difficult for archaeologists to establish the purpose of this important tool found in many different locations around the world. Nowell and Chang (2009) review the available ethological and archaeological evidence and provide a detailed critique of Kohn and Mithen's explanation. In accord with the analysis of this paper they conclude that

"the evidence strongly suggests that variation in handaxe morphology is governed by a number of complex factors that differ in influence over time and space, rather than a single overarching mechanism such as sexual selection. As with most questions of interest in Palaeolithic archaeology, we are better served by context-specific, historically situated explanations rather than monocausal scenarios, particularly when our desire is to understand the form and function of an artefact such as the handaxe, which is found in the record, in one form or another, for more than 1.5 million years" (p. 84).

The foregoing considerations suggest that an evolutionary explanation of conspicuous consumption is not well supported by historical evidence. It is more plausible to argue that conspicuous consumption is a kind of behavior undertaken in a social context that already includes aspects such as rapid population growth, high population densities, technological innovations, political institutions and formalized hierarchies based on the inheritance of wealth. Human societies grew in complexity due to labor specialization and notable inequalities in the production and the consumption of resources began to emerge. Moreover, production lost its privileged position and consumption became the mean through which individuals present themselves to others.

This proposal is explicit in Veblen's work. In first chapters of the Theory of the Leisure Class, Veblen (1899) described the social and historical background of consumption phenomena. In contrast to contemporary research on conspicuous consumption, Veblen's analysis was based on the anthropological work of his time. This allowed him to distinguish modern market societies from traditional societies that lived at the edge of subsistence struggling to acquire the basic means for survival. ${ }^{7}$ Without references to particular historical periods, Veblen positioned conspicuous consumption within a particular social and cultural context. He examined the display of commodities as a mean of securing social differentiation and status in societies characterized by property rights and the accumulation of monetary and material resources.

Apart from distinguishing between the modern and ancestral societies, Veblen's second important contribution is that he analyzed evolved psychological aspects of

\footnotetext{
${ }^{7}$ He summarizes his views in the following way: "They are small groups and of a simple (archaic) structure; they are commonly peaceable and sedentary; they are poor; and the individual ownership is not a dominant feature of their economic system." (Veblen 1899: p.8)
} 
human conspicuous consumption. He argued that "irreducible elements of human nature", what he called "instincts", might have a significant influence on evolutionary processes (1914, p.3). According to Richerson and Christiansen (2013), Veblen's work on human predispositions "clearly foreshadows the notion of epigenetic rules, cultural selection, and biased transmission that figure in the late twentieth century revival of Darwinian theories of cultural evolution" (p.7). In the section that follows I will expand on this insight to present an alternative explanation of conspicuous consumption.

\section{Social learning and conspicuous consumption}

In the previous sections I argued that an evolutionary explanation of conspicuous consumption based on sexual selection and costly signaling theory faces conceptual and empirical problems. First, it is not clear what kind of traits researchers have in mind by conspicuous consumption. Second, conspicuous consumption does not appear to be a costly signaling trait. Finally, the available evidence from huntergatherer societies casts doubt on whether conspicuous consumption evolved as a mating strategy.

Given that the long term evolution of conspicuous consumption is difficult to explain on the basis of sexual selection and costly signaling theory, it is worth exploring alternative theoretical accounts. In line with Veblen's work, my suggestion in the previous section was that conspicuous consumption is better conceived as a type of overt behavior that is present in contemporary market economies. In contrast to an evolutionary explanation based on costly signaling theory that applies Darwinian theory directly to conspicuous consumption, what figures prominently in the account advanced in this section is that biological evolution has built a set of cognitive adaptations in ancestral environments that have a lasting influence on economic behavior (e.g. Witt 2008).

A key question is what are the cognitive foundations of conspicuous consumption? One way to answering this question is to apply cultural evolution theory, the most advanced theoretical approach to studying social learning mechanisms and the dynamics of cultural change (e.g. Boyd and Richerson 1985, Richerson and Boyd 2005). In what follows, I will explore more in depth areas of consumption research where cultural evolution can provide an original contribution.

According to Richerson and Boyd (2005), extensive and cumulative cultural evolution is the main reason that modern societies are different from ancestral societies. From an evolutionary perspective, "culture" refers to ideas, skills, beliefs, habits, behaviors and values transmitted from one individual to another via imitation, teaching and other forms of social learning (Boyd and Richerson, 1985, Henrich and McElreath 2003, Richerson and Boyd 2005). This definition of culture emphasizes the importance of cognitive abilities to acquire behaviors, ideas etc. by observing or interacting with others. For example, a child that reaches puberty may start to imitate cultural traits of her teachers or her friends and abandon traits acquired from her parents. This can be the result of peer pressure or personal experience that made her realize that parent imitation is not useful anymore. 
Various kinds of cognitive biases structure human social learning and the transmission of behaviors, ideas, and other components of culture. These biases can be understood as individual level mechanisms or as population-level properties that affect the rate of transmission within and between different groups. Cultural evolution theory typically distinguishes the following types of learning biases:

i) Content bias (or "direct" bias) is similar to economists' cost-benefit analysis. An individual selects a trait based on its perceived value or intrinsic attractiveness.

ii) Frequency-dependent biases refer to the relationship between the frequency of a trait and its probability of adoption. Individuals may put higher or lower weight to more frequent traits (conformity bias and anti-conformity bias).

iii) Context bias (or "indirect" bias) refers to individuals acquiring traits based on the characteristics of an individual (or "model") who exhibits it. Social learners use a cue such as age, gender, familiarity, prestige and success in a particular domain to determine whether a particular individual is an appropriate model.

In line with this research, it is possible to suggest that different social learning biases are involved in conspicuous consumption.

First, according to content bias, individuals acquire consumption behaviors by observing and comparing their main characteristics to other alternatives. They may consume specific goods simply because they think that some of their features (their content) are better than other goods. Even if some goods are purchased and consumed primarily for their display value, most of them have also use value. Consumers may acquire conspicuous goods because of their speed, design or comfort. To use an example from the literature, a consumer may buy a Porsche car instead of a VW car because it allows her to move fast from one location to another with relative safety.

Second, according to frequency-dependent biases, individuals have the propensity to adopt consumption behaviors that are common or rare in the population. Although future empirical research might test which of the two biases - conformity or anti-conformity - is more potent in the case of conspicuous consumption, both provide insights into human consumption behavior. In particular, consumers are disproportionately more likely to copy behaviors that are present at a high frequency to conform to the majority of the group or the population they belong. Acquiring expensive goods to communicate a conforming image is often necessary to gain acceptance by other group members. In the case of conspicuous consumption this motivation appears to be more significant and pronounced. Hence, the intuitive suggestion of cultural evolution theory is that independently of their position in a social hierarchy, individuals have a propensity to conform to the expectations of groups with whom they are associated with and this limits the variability of individual consumer behavior within these groups.

Conversely, consumers might have the propensity to differ from the consumption behaviors that are most common in the population or a group. ${ }^{8}$ One key characteristic of conspicuous consumption is exclusivity - consumers desire to be perceived

\footnotetext{
${ }^{8}$ In economics this is described by Leibenstein (1950) as the "snob effect."
} 
as different from the members of the group they belong or the population in general. Hence, goods that are considered scarce or unique appear to have higher value. Moreover, the perceived exclusivity of particular goods decreases when more consumers purchase and demonstrate them in public. To return to the Porsche example, although there is a long waiting list for Porsche cars, in order to maintain their sales, Porsche limits the quantity of cars produced by their factories to a few thousand vehicles per year. This is also the case with goods such as watches, jewellery, perfumes or collector's items such as stamps. Producers of consumption goods highlight their exclusivity since many consumers would find a Porsche less valuable if it is widely available. An insight of cultural evolution theory compared to sexual selection is that rather than to display their wealth, social status or particular quality traits to potential mates, consumers would often choose to buy and display expensive goods just for the sake of being different from other consumers.

Third, consumers may have a propensity to acquire specific consumption behaviors based on the observable attributes of a model individual that exhibits particular values, ideas and behavior that are considered important within a group. Veblen (1899) was one of the first theorists who argued that consumption is actually a process of emulation and goods function as markers of model individuals. According to this idea, individuals emulate the consumption patterns of those that are perceived as higher in the hierarchy of a group or the population. However, in modern times, emulation is a round or two-directional process: it can be top-down or bottom-up. According to Trigg (2001), Bourdieu $(1984,1990)$ provides a contemporary development of the theory of conspicuous consumption. Although consumers typically emulate the consumption pattern of prosperous or prestigious individuals, Bourdieu's hypothesis is that emulation can also be bottom-up: those with a higher position in the social hierarchy might emulate the behavior and consumption patterns of those in a lower position. Individuals with a higher status may increase their reliance on social learning and copy those who they perceive as having lower status because they see them as superior sources of information.

What reason do we have for thinking, on the basis of empirical work, that such psychological biases are present and influence conspicuous consumption? Evolutionary explanations are more plausible when they are supported by empirical evidence from multiple sources. Laboratory experiments and field studies have provided evidence for many of the biases explored in the theoretical literature and resonate with an explanation of conspicuous consumption based on social learning biases. There is good evidence that conformist and anti-conformist learning is a feature of our psychology and has a strong influence on our behavior (e.g. McElreath et al. 2005, Morgan and Laland 2012). Moreover, experimental research suggests that under controlled conditions children selectively learn a wide range of traits from prestigious and successful individuals (e.g. Chudek et al. 2012). ${ }^{9}$ Consistent with this, ethnographic research that focuses on how children of hunter-gatherer groups learn different hunting and gathering skills and whom they learn from aligns with

9 There are concerns about importing results from empirical and theoretical psychological work into cultural evolution theory. Building on insights from social psychology requires detailed conceptual analysis (e.g. Lewens, 2015). 
cultural evolution theory (e.g. MacDonald 2007, Lew-Levy et al. 2017). In domains such as fishing, growing yams and using medicinal plants, prestige and success guide model-based bias among people living on the Yasawa Island of the Fijian Archipelago (Henrich and Broesh 2011). Moreover, the presence of prestige-bias social learning of beneficial food taboos is documented in traditional Fijian populations (Henrich and Henrich 2010).

Evolutionary researchers might raise the following objection. The analysis in this section refers to proximate behavior-generating mechanisms, not evolutionary causes such as sexual selection. My response is based on Henrich and McElreath's (2007) work on the evolution of social learning biases:

- Genetic evolution gives rise to psychological adaptations that allow the acquisition of information such as ideas, beliefs or strategies of other individuals.

- These adaptations allow the evolution of the cultural system of inheritance independent from genetic inheritance. Humans become gradually reliant on social learning and cultural evolution could arise.

- Cultural evolution processes might change the selection conditions on which natural selection operates.

Evolutionary approaches suggest that our species' social learning biases - the psychological mechanisms that underlie the evolution of traits such as conspicuous consumption - are genetically evolved adaptations in ancestral environments. It is social learning mechanisms that evolved to enhance fitness while conspicuous consumption taps into psychological biases already in place. Put in a different way, social learning biases arose some time during our evolutionary history because they were favored by natural selection and have remained an important feature of human psychology since that time. Hence the question that needs to be addressed is whether genetic evolution gave rise to psychological adaptations that have an influence on conspicuous consumption.

In the last 30 years, evolutionary theorists have produced a large number of evolution models that examine the dynamics of learning biases. Within the framework of cultural evolution, formal work explores environmental conditions that favor the evolution of social learning strategies (e.g. Boyd and Richerson, 1985, Henrich and Boyd, 1998, Rendell et al. 2009, Nakahashi et al. 2012). In particular, theoretical research predicts under what conditions social learning biases and individual learning evolve and whether it is evolutionarily advantageous to use multiple social learning biases. It is argued that if environments did not change rapidly and the rate of migration between groups was low, conformist or prestige bias would have been favored over individual learning. However, in rapidly changing environments, social transmission will be of little benefit since the information accumulated from past generations would be quickly outdated. In such environments, individual learners that experiment with alternative traits will perform better since they acquire information directly from their environment.

To sum up, I put forward an account of conspicuous consumption based on the evolution of human social learning biases. Conspicuous consumption can be traced back to evolved cognitive biases that shape what we learn and whom we learn from. Moreover, the evolutionary explanation presented in this section is plausible from the perspective of natural selection. However, consistency of this account with existing evidence is not 
equivalent to establishing that conspicuous consumption resulted from social learning adaptations. The work in the previous paragraphs takes seriously the task of giving some initial support to an evolutionary explanation of conspicuous consumption based on cultural evolution theory. It can serve a valuable heuristic role throughout evolutionary economics by setting out the foundations for further research. But it is far from being conclusive or complete. But the following issues remain when modern cultural evolution theory is applied to formulate an evolutionary explanation of conspicuous consumption.

First, evolutionary researchers take inspiration from population genetics and social psychology to build evolutionary models that emphasize particular social learning biases. However, there has been very little formal modeling of how social learning influences the evolution of display traits such as conspicuous consumption. As far as I am aware of there is only one rigorous attempt that carefully examines the evolution of conspicuous consumption (Boyd and Richerson, 1985: ch.8, for a discussion see Cordes 2009). Boyd and Richerson demonstrate that a runaway process can lead a display trait such as conspicuous consumption to take exaggerated forms. Unfortunately, conspicuous consumption is relatively infrequent (or entirely unexplored) subjects of analysis using Darwinian concepts and methods. Although psychological propensities can be easily modeled as frequency-dependent selection strategies, there is no formal work that examines the role of conformity and anticonformity transmission to the emergence and stability of conspicuous consumption.

Second, the previous discussion suggests that empirical research causes trouble for an evolutionary account based on sexual selection and costly signaling theory and that an explanation of conspicuous consumption based on social learning appears to fit with available empirical evidence. However, one should not be too hasty to conclude that this evolutionary explanation is well supported. To make this explanation much more plausible, evolutionary researchers need to link this explanation to fine grained empirical data from archaeological and palaeoanthropological records. Evolutionary models and simulations demonstrate what sort of social learning biases would have evolved under a very broad range of plausible environmental conditions. However, empirical data about longrun dynamics of cultural transmission mechanisms are rare. To improve our understanding of conspicuous consumption, a theoretical reflection on the selection conditions in ancestral environments must be supported by detailed historical evidence.

Having said this, applying cultural evolutionary theory has important advantages. First, cultural evolution theory provides the mathematical tools to build evolutionary models that link social learning biases to population-level dynamics. Another advantage is that it is possible to provide a taxonomy that collects diverse concrete factors that produce conspicuous consumption into classes with similar features. The processes of selectively acquiring particular traits are attributed to different forms of cognitive biases. Finally, whereas the evolution of cognitive biases must be studied using evidence from the ancestral environment, the influence of these biases on consumption behavior is empirically accessible to historical observation in modern societies. Thus, it is easier to identify and subsequently empirically examine what generates conspicuous consumption. Hence, cultural evolution theory's commitment to develop evolutionary explanations informed by cognitive science, evolutionary theory and evidence about the selection environment can provide novel insights into the evolution of human economic behavior. 


\section{Conclusion}

This paper explores the evolution of what is commonly referred to as conspicuous consumption in ancestral and contemporary societies. With respect to sexual selection and costly signaling theory this paper leads to the conclusion that the study of conspicuous consumption in human primates needs a critical examination of the assumptions made and the data gathered. The available data demonstrate that our ancestors lived in small, relatively stable, egalitarian groups and therefore it is doubtful that conspicuous consumption is a sexual adaptation. Females were likely to accumulate information about potential mates from multiple sources over a long time frame.

After examining the hypothesis that conspicuous consumption is a sexual adaptation, I suggest that whereas human psychological capacities may have a long history in the human lineage, conspicuous consumption appears relatively late human history. Cultural evolution theory can elucidate evolved aspects of our psychology that promote and maintain conspicuous consumption in the population.

Explaining the complex phenomenon of conspicuous consumption is still not complete. The exact nature of human cognition involved in conspicuous consumption has not been fully investigated and understanding the underlying psychology remains a largely open issue. Future studies have to examine the psychological and evolutionary side of the issue and evaluate cultural and ecological factors that influence conspicuous consumption.

Acknowledgements Earlier versions benefited from the comments of Jack Vromen, Krist Vaesen, Oskar van Eeden and participants of seminars at Erasmus Institute for Philosophy and Economics and the Philosophy and Ethics workshop at Eindhoven University of Technology. The usual caveat applies.

Availability of data and material Not applicable.

Code availability Not applicable.

\section{Declarations}

Conflicts of interest/competing interests Not applicable.

Open Access This article is licensed under a Creative Commons Attribution 4.0 International License, which permits use, sharing, adaptation, distribution and reproduction in any medium or format, as long as you give appropriate credit to the original author(s) and the source, provide a link to the Creative Commons licence, and indicate if changes were made. The images or other third party material in this article are included in the article's Creative Commons licence, unless indicated otherwise in a credit line to the material. If material is not included in the article's Creative Commons licence and your intended use is not permitted by statutory regulation or exceeds the permitted use, you will need to obtain permission directly from the copyright holder. To view a copy of this licence, visit http://creativecommons.org/ licenses/by/4.0/.

\section{References}

Aoki K, Nakahashi W (2008) Evolution of learning in subdivided populations that occupy environmentally heterogeneous sites. Theor Popul Biol 74:356-368. https://doi.org/10.1016/j.tpb.2008.09.006 
Bliege Bird R, Smith EA (2005) Signaling theory, strategic interaction, and symbolic capital. Curr Anthropol 46:221-248. https://doi.org/10.1086/427115

Bourdieu P (1984) Distinction: a social critique of the judgement of taste: Pierre Bourdieu. Harvard University Press, Cambridge MA

Bourdieu P (1990) The logic of practice. Stanford University Press, Stanford CA

Bowles S, Gintis H (2011) A cooperative species: human reciprocity and its evolution. Princeton University Press, Princeton

Boyd R, Richerson PJ (1985) Culture and the evolutionary process. University of Chicago Press, Chicago

Buss D (1989) Sex differences in human mate preferences: evolutionary hypotheses tested in 37 cultures. Behav Brain Sci 12:1-14. https://doi.org/10.1017/s0140525x00023992

Buss D (2016) The evolution of desire: strategies of human mating. Basic Books, New York

Chudek M, Heller S, Birch S, Henrich J (2012) Prestige-biased cultural learning: bystander's differential attention to potential models influences children's learning. Evol Hum Behav 33:46-56. https://doi. org/10.1016/j.evolhumbehav.2011.05.005

Chudek M, Muthukrishna M, Henrich J (2016) Cultural evolution. In: Buss D (ed) The handbook of evolutionary psychology: integrations. John Wiley \& Sons, New Jersey, pp 749-769. https://doi.org/10. 1002/9781119125563.evpsych230

Collins J, Baer B, Weber EJ (2015) Sexual selection, conspicuous consumption and economic growth. J Bioecon 17:189-206. https://doi.org/10.1007/s10818-015-9200-9

Cordes C (2009) The role of biology and culture in Veblenian consumption dynamics. J Econ Issues 43:115-142. https://doi.org/10.2753/jei0021-3624430106

Darwin, C. (1871/1901) The descent of man and selection in relation to sex. J. Murray, London

Dezecache G, Mercier H, Scott-Phillips TC (2013) An evolutionary approach to emotional communication. J Pragmati 59:221-233. https://doi.org/10.1016/j.pragma.2013.06.007

Efferson C, Lalive R, Richerson P, Mcelreath R, Lubell M (2008) Conformists and mavericks: the empirics of frequency-dependent cultural transmission. Evol and Hum Behav 29:56-64. https://doi.org/ 10.1016/j.evolhumbehav.2007.08.003

Ember CR (1978) Myths about hunter-gatherers. Ethnology 17:439. https://doi.org/10.2307/3773193

Fraja GD (2009) The origin of utility: sexual selection and conspicuous consumption. J Econ Behav Organ 72:51-69. https://doi.org/10.1016/j.jebo.2009.05.019

Gamble C (1999) The Palaeolithic societies of Europe. Cambridge University Press, Cambridge

Geary DC (1998) Male, female: the evolution of human sex differences. American Psychological Association, Washington DC

Gould SJ, Lewontin RC (1979) The spandrels of san Marco and the Panglossian paradigm: a critique of the adaptationist programme. Proc R Soc London B Biol Sci 205:581-598. https://doi.org/10.1098/ rspb. 1979.0086

Grafen A (1990a) Biological signals as handicaps. J Theor Biol 144:517-546. https://doi.org/10.1016/ s0022-5193(05)80088-8

Grafen A (1990b) Sexual selection unhandicapped by the fisher process. J Theor Biol 144:473-516. https://doi.org/10.1016/s0022-5193(05)80087-6

Gray P (2014) Play theory of hunter-gatherer egalitarianism. Ancestral Landscapes in Human Evolution:192-215. https://doi.org/10.1093/acprof:oso/9780199964253.003.0014

Griskevicius V, Tybur JM, Sundie JM, Cialdini RB, Miller GF, Kenrick DT (2007) Blatant benevolence and conspicuous consumption: when romantic motives elicit strategic costly signals. J Pers Soc Psychol 93:85-102. https://doi.org/10.1037/0022-3514.93.1.85

Grose J (2011) Modelling and the fall and rise of the handicap principle. Biol Philos 26:677-696. https:// doi.org/10.1007/s10539-011-9275-1

Henrich J, Boyd R (1998) The evolution of conformist transmission and the emergence of between-group differences. Evol Hum Behav 19:215-241. https://doi.org/10.1016/s1090-5138(98)00018-x

Henrich J, Gil-White FJ (2001) The evolution of prestige: freely conferred deference as a mechanism for enhancing the benefits of cultural transmission. Evol and Hum Behav 22:165-196. https://doi.org/ 10.1016/s1090-5138(00)00071-4

Henrich J, McElreath R (2003) The evolution of cultural evolution. Evol Anthropol Issues News Rev 12:123-135. https://doi.org/10.1002/evan.10110

Henrich J, McElreath R (2007) Dual-inheritance theory: the evolution of human cultural capacities and cultural evolution. Oxford University Press. https://doi.org/10.1093/oxfordhb/9780198568308.013.0038

Henrich J, Henrich N (2010) The evolution of cultural adaptations: Fijian food taboos protect against marine toxins. Proc R Soc B 277:3715-3724. https://doi.org/10.1098/rspb.2010.1191 
Henrich J and Broesch J (2011) On the nature of cultural transmission networks: evidence from Fijian villages for adaptive learning biases Phil trans R Soc B 366: 1139-1148. https://doi.org/10.1098/ rstb.2010.0323

Kelly RL (2007) The foraging spectrum: diversity in hunter-gatherer lifeways. Percheron Press, New York

Kohn M, Mithen S (1999) Handaxes: products of sexual selection? Antiquity 73:518-526. https://doi.org/ $10.1017 / \mathrm{s} 0003598 \times 00065078$

Kurland JA (2001) The mating mind: how sexual choice shaped the evolution of the human mind. Am Anthropol 103:1196-1198. https://doi.org/10.1525/aa.2001.103.4.1196

Lee RB (2009) The!Kung san: men, women, and work in a foraging society. Cambridge University Press, Cambridge

Leibenstein H (1950) Bandwagon, snob, and Veblen effects in the theory of consumers demand. Q J Econ 64:183. https://doi.org/10.2307/1882692

Lewens T (2015) Cultural evolution: conceptual challenges. Oxford University Press, Oxford

Lew-Levy S, Reckin R, Lavi N et al (2017) How do hunter-gatherer children learn subsistence skills? Hum Nat 28:367-394. https://doi.org/10.1007/s12110-017-9302-2

MacDonald K (2007) Cross-cultural comparison of learning in human hunting. Hum Nat 18:386-402. https://doi.org/10.1007/s12110-007-9019-8

Marlowe FW (2005) Hunter-gatherers and human evolution. Evol Anthropol Issues News Rev 14:54-67. https://doi.org/10.1002/evan.20046

McElreath R, Henrich J (2007) Modelling cultural evolution. Oxford University Press. https://doi.org/10. 1093/oxfordhb/9780198568308.013.0039

McElreath R, Lubell M, Richerson PJ, Waring TM, Baum W, Edsten E, Efferson C, Paciotti B (2005) Applying evolutionary models to the laboratory study of social learning. Evol Hum Behav 26:483508. https://doi.org/10.1016/j.evolhumbehav.2005.04.003

Miller G (2001) The mating mind: how sexual choice shaped the evolution of human nature. Vintage, London

Miller G (2009) Spent sex, evolution, and consumer behavior. Penguin Group Usa, New York

Morgan TJ, Laland KN (2012) The biological bases of conformity. Front Neurosci 6:87. https://doi.org/ 10.3389/fnins.2012.00087

Nakahashi W, Wakano JY, Henrich J (2012) Adaptive social learning strategies in temporally and spatially varying environments. Hum Nat 23:386-418. https://doi.org/10.1007/s12110-012-9151-y

Nelissen RM, Meijers MH (2011) Social benefits of luxury brands as costly signals of wealth and status. Evol Hum Behav 32:343-355. https://doi.org/10.1016/j.evolhumbehav.2010.12.002

Nowell, A, Chang ML (2009) The case against sexual selection as an explanation of handaxe morphology. Paleoanthropology: 77-88. Retrieved from http://www.paleoanthro.org/static/journal/content/ PA20090077.pdf

Rendell L, Fogarty L, Hoppitt WJE, Morgan TJ, Webster MM, Laland KN (2011) Cognitive culture: theoretical and empirical insights into social learning strategies. Trends in Cognit Sci 15:68-76. https://doi.org/10.1016/j.tics.2010.12.002

Richerson PJ, Boyd R (2005) Not by genes alone: how culture transformed human evolution. The University of Chicago Press, Chicago

Richerson PJ, Christiansen MH (2013) Cultural evolution: society, technology, language and religion. MIT Press, Cambridge, MA

Saad G (2014) The evolutionary bases of consumption. Psychology Press, New York

Smith JM, Harper D (2003) Animal signals. Oxford University Press, Oxford

Sundie JM, Kenrick DT, Griskevicius V, Tybur JM, Vohs KD, Beal DJ (2011) Peacocks, Porsches, and Thorstein Veblen: conspicuous consumption as a sexual signaling system. J Pers Soc Psychol 100:664-680. https://doi.org/10.1037/a0021669

Számadó S (2011) The cost of honesty and the fallacy of the handicap principle. Anim Behav 81:3-10. https://doi.org/10.1016/j.anbehav.2010.08.022

Trigg AB (2001) Veblen, Bourdieu, and Conspicuous Consumption. J Econ Issues 35:99-115. https://doi. org/10.1080/00213624.2001.11506342

Veblen T (1914) The instinct of workmanship and the state of the industrial arts. W. W. Norton And Company, New York

Veblen T (1970) The theory of the leisure class: an economic study of institutions. Allen And Unwin, London

Wakano JY, Aoki K (2007) Do social learning and conformist bias coevolve? Henrich and Boyd revisited. Theor Popul Biol 72:504-512. https://doi.org/10.1016/j.tpb.2007.04.003

Witt U (2008) What is specific about evolutionary economics? J Evol Econ 18:547-575. https://doi.org/ 10.1007/s00191-008-0107-7 
Witt U (2017) The evolution of consumption and its welfare effects. J Evol Econ 27:273-293. https://doi. org/10.1007/s00191-016-0459-3

Woodburn J (1982) Egalitarian Societies. Man 17(3):431. https://doi.org/10.2307/2801707

Zahavi A (1975) Mate selection-a selection for a handicap. J Theor Biol 53:205-214. https://doi.org/10. 1016/0022-5193(75)90111-3

Publisher's note Springer Nature remains neutral with regard to jurisdictional claims in published maps and institutional affiliations. 